\title{
Multiple Solutions to Fractional Difference Boundary Value Problems
}

\author{
Huiqin Chen, Yaqiong Cui, and Xianglan Zhao \\ School of Mathematics and Computer Sciences, Shanxi Datong University, Datong, Shanxi 037009, China \\ Correspondence should be addressed to Huiqin Chen; dtdxchq@126.com
}

Received 7 February 2014; Revised 6 April 2014; Accepted 6 April 2014; Published 27 April 2014

Academic Editor: Mohamed Boussairi Jleli

Copyright (c) 2014 Huiqin Chen et al. This is an open access article distributed under the Creative Commons Attribution License, which permits unrestricted use, distribution, and reproduction in any medium, provided the original work is properly cited.

\begin{abstract}
The following fractional difference boundary value problems $\Delta^{v} y(t)=-f(t+v-1, y(t+v-1)), y(\nu-2)=y(\nu+b+1)=0$ are considered, where $1<v \leq 2$ is a real number and $\Delta^{v} y(t)$ is the standard Riemann-Liouville fractional difference. Based on the Krasnosel'skiì theorem and the Schauder fixed point theorem, we establish some conditions on $f$ which are able to guarantee that this FBVP has at least two positive solutions and one solution, respectively. Our results significantly improve and generalize those in the literature. A number of examples are given to illustrate our main results.
\end{abstract}

\section{Introduction}

Fractional difference equations have been of great interest recently. It is caused by the intensive development of the theory of discrete fractional calculus itself; see [1-8]. Diaz and Osler [1] introduced a fractional difference defined as an infinite series, a generalization of the binomial formula for the $N$ th order difference $\Delta^{N} f$. Gray and Zhang [2] developed a special case for one composition rule and Leibniz formula. They worked exclusively with the nabla operator. A recent interest in discrete fractional calculus has been shown by Atici et al.; see [3-12]. Atici and Eloe developed some of the basic theory of both discrete fractional IVPs and BVPs with delta derivative on the time scale $\mathbb{Z}$. In particular, Atıcı and Şengül [5] provided some analysis of discrete fractional variational problems. Their paper also provided some initial attempts at using the discrete fractional calculus to model biological processes. Similarly, Goodrich [7-12] has established some results on both discrete fractional IVPs and BVPs. Holm [13] introduced fractional sum and difference operators and presented a complete and precise theory for composing fractional sums and differences. In addition, $\mathrm{Wu}$ and Baleanu [14] mainly concentrated on the analytical aspects, and the variational iteration method is extended in a new way to solve an initial value problem of $q$-fractional difference equations. Following this trend, in $[15,16]$, the authors discussed the boundary value problems of fractional difference equations depending on parameters.

In this paper, we consider the following boundary value problems for a fractional difference equation (FBVP):

$$
\begin{gathered}
\Delta^{v} y(t)=-f(t+v-1, y(t+v-1)), \\
y(v-2)=y(v+b+1)=0,
\end{gathered}
$$

where $t \in[0, b+1]_{\mathbb{N}_{0}}, f:[\nu-1, v+b]_{\mathbb{N}_{\nu-1}} \times \mathbb{R} \rightarrow \mathbb{R}$ is continuous and $f$ is not identically zero, $1<v \leqslant 2, b>2$ is an integer, and $\Delta^{v} y(t)$ is the standard Riemann-Liouville fractional difference. In this paper, we will use properties of Green's function of the FBVP (1) and the Krasnosel'skii fixed point theorem to show that the FBVP (1) has at least one or two positive solutions. Our results significantly improve and generalize the results in $[6,8]$.

The plan of this paper is as follows. In Section 2, we will present some necessary lemmas. By using the Krasnosel'skiǐ theorem, Section 3 proves the existence of two positive solutions for the FBVP (1). Section 4 deduces the existence of one solution by using Schauder's fixed point theorem.

\section{Preliminaries}

In this section, we first review some basic notations and lemmas about fractional sums and differences in $[6-8,13]$. 
For any $t$ and $\nu$, we define

$$
t^{\underline{\nu}}=\frac{\Gamma(t+1)}{\Gamma(t+1-v)}
$$

for which the right-hand side is defined. We appeal to the convention that if $t+1-v$ is a pole of the Gamma function and $t+1$ is not a pole, then $t^{\underline{\nu}}=0$.

The $v$ th fractional sum of a function $f$ is

$$
\Delta^{-v} f(t)=\frac{1}{\Gamma(\nu)} \sum_{s=a}^{t-v}(t-s-1)^{\frac{\nu-1}{}} f(s)
$$

for $\nu>0$ and $t \in\{a+v, a+v+1, \ldots\}=\mathbb{N}_{a+v}$. We also define the $\nu$ th fractional difference for $\nu>0$ by $\Delta^{\nu} f(t)=\Delta^{n} \Delta^{-(n-\nu)} f(t)$, where $t \in \mathbb{N}_{a+n-\gamma}$, and $n \in \mathbb{N}$ is chosen such that $0 \leqslant n-1<$ $v \leqslant n$.

$$
\begin{aligned}
& \text { Let } 0 \leqslant n-1<v \leqslant n \text {. Then } \\
& \begin{aligned}
\triangle^{-v} \triangle^{\nu} f(t)= & f(t)+c_{1}(t-a)^{\frac{\nu-1}{}}+c_{2}(t-a)^{\frac{\nu-2}{2}} \\
& +\cdots+c_{n}(t-a)^{\frac{\nu-n}{2}}, \quad c_{i} \in \mathbb{R}, \quad 1 \leqslant i \leqslant n .
\end{aligned}
\end{aligned}
$$

In order to prove our results, we now provide some properties on Green's function associated with the problem (1).

Lemma 1 (see [6, Theorem 3.1]). Let $1<v \leqslant 2$ and $f:[v-$ $1, v+b]_{\mathbb{N}_{\nu-1}} \times \mathbb{R} \rightarrow \mathbb{R}$ be given. Then the solution of the FBVP (1) is given by

$$
y(t)=\sum_{s=0}^{b+1} G(t, s) f(s+v-1, y(s+v-1)),
$$

where Green's function $G:[\nu-1, \nu+b]_{\mathbb{N}_{\nu-1}} \times[0, b+1]_{\mathbb{N}_{0}} \rightarrow \mathbb{R}$ is defined by

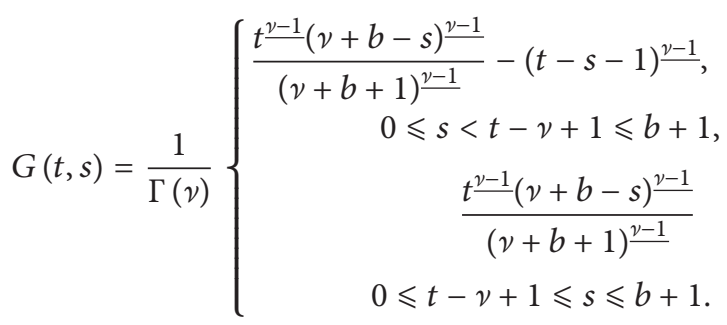

Remark 2. It is easy to see that $G(\nu-2, s)=G(\nu+b+1, s)=$ 0 . $G$ could be extended to $[\nu-2, v+b+1]_{\mathbb{N}_{\nu-1}} \times[0, b+1]_{\mathbb{N}_{0}}$; we only discuss $(t, s) \in[\nu-1, v+b]_{\mathbb{N}_{\nu-1}} \times[0, b+1]_{\mathbb{N}_{0}}$.

Lemma 3 (see [6, Theorem 3.2]). The Green function $G(t, s)$ satisfies the following conditions.

(i) $G(t, s)>0,(t, s) \in[\nu-1, v+b]_{\mathbb{N}_{v-1}} \times[0, b+1]_{\mathbb{N}_{0}}$.

(ii) $\max _{t \in[\nu-1, v+b]_{\mathbb{N}_{\nu-1}}} G(t, s)=G(s+v-1, s), s \in[0, b+1]_{\mathbb{N}_{0}}$.

(iii) There exists a number $\gamma \in(0,1)$ such that

$$
\begin{gathered}
\min _{(\nu+b) / 4 \leqslant t \leqslant 3(\nu+b) / 4} G(t, s) \geqslant \gamma \max _{t \in[\nu-1, \nu+b]_{\mathbb{N}_{\nu-1}}} G(t, s) \\
=\gamma G(s+\nu-1, s), \quad s \in[0, b+1]_{\mathbb{N}_{0}} .
\end{gathered}
$$

Denote

$$
\begin{array}{r}
\mathscr{B}=\left\{y:[v-2, v+b+1]_{\mathbb{N}_{\nu-2}} \longrightarrow \mathbb{R},\right. \\
y(\nu-2)=y(\nu+b+1)=0\} .
\end{array}
$$

It is clear that $\mathscr{B}$ is a Banach space with the norm $\|y\|=$ $\max _{t \in[v-2, v+b]_{\mathbb{N}_{0}}}|y(t)|$. We choose a cone

$$
\mathscr{K}=\left\{y \in \mathscr{B}: y(t) \geqslant 0, \min _{(\nu+b) / 4 \leqslant t \leqslant 3(\nu+b) / 4} y(t) \geqslant \gamma\|y\|\right\} .
$$

Now consider the operator $T$ defined by

$$
(T y)(t)=\sum_{s=0}^{b+1} G(t, s) f(s+v-1, y(s+v-1)) .
$$

Referring to Lemma 3.1 of [8], we have the following.

\section{Lemma 4.}

$$
T(\mathscr{K}) \subseteq \mathscr{K}
$$

We notice that $T$ is a summation operator on a discrete finite set. Hence, $T$ is trivially completely continuous. And a fixed point of $T$ is equivalent to a solution of the FBVP (1). We will obtain sufficient conditions on the nonlinear $f$ for the existence of fixed points of $T$. In order to prove our results, we need the following well-known Krasnosel'skiǐ fixed point theorem.

Lemma 5 (see [17]). Let $\mathscr{B}$ be a Banach space and let $\mathscr{K} \subseteq$ $\mathscr{B}$ be a cone. Assume that $\Omega_{1}$ and $\Omega_{2}$ are bounded open sets contained in $\mathscr{B}$ such that $0 \in \Omega_{1}$ and $\bar{\Omega}_{1} \subseteq \Omega_{2}$. Assume $T$ : $\mathscr{K} \cap\left(\bar{\Omega}_{2} \backslash \Omega_{1}\right) \rightarrow \mathscr{K}$ is a completely continuous operator. If either

(i) $\|T y\| \leqslant\|y\|$ for $y \in \mathscr{K} \cap \partial \Omega_{1}$ and $\|T y\| \geqslant\|y\|$ for $y \in \mathscr{K} \cap \partial \Omega_{2} ;$ or

(ii) $\|T y\| \geqslant\|y\|$ for $y \in \mathscr{K} \cap \partial \Omega_{1}$ and $\|T y\| \leqslant\|y\|$ for $y \in \mathscr{K} \cap \partial \Omega_{2}$.

Then the operator $T$ has at least one fixed point in $\mathscr{K} \cap\left(\bar{\Omega}_{2} \backslash \Omega_{1}\right)$.

\section{Existence of Positive Solutions}

In this section, we state and prove the multiplicity of positive solutions regarding FBVP (1). Then, we conclude this section with two examples to illustrate our main results. For this, we 
need to suppose that $f:[\nu-1, \nu+b]_{\mathbb{N}_{\nu-1}} \times[0,+\infty) \rightarrow[0,+\infty)$ is continuous and $f$ is not identically zero. Denote

$$
\begin{aligned}
f_{0} & =\liminf _{y \rightarrow 0} \min _{t \in[\nu-2, \nu+b]_{\mathbb{N}-2}} \frac{f(t, y)}{y}, \\
f^{0} & =\limsup _{y \rightarrow 0} \max _{t \in[\nu-2, v+b]_{\mathbb{N}-2}} \frac{f(t, y)}{y}, \\
f_{\infty} & =\liminf _{y \rightarrow+\infty} \min _{t \in[\nu-2, v+b]_{N_{\nu-2}}} \frac{f(t, y)}{y}, \\
f^{\infty} & =\limsup _{y \rightarrow+\infty} \max _{t \in[\nu-2, \nu+b]_{\mathbb{N}-2}} \frac{f(t, y)}{y}, \\
\eta & =\frac{1}{\sum_{s=0}^{b+1} G(s+\nu-1, s)}, \\
\lambda & =\frac{1}{\gamma \sum_{s=[(\nu+b) / 4-\nu+1]}^{[3(\nu+b) / 4-\nu+1]} G([(b-v) / 2]+\nu, s)},
\end{aligned}
$$

where $\gamma$ is the constant in Lemma 3. In the sequel, let $\Omega_{r}=$ $\{y \in \mathscr{K}:\|y\|<r\}$, for $r>0$, and $\partial \Omega_{r}=\{y \in \mathscr{K}:\|y\|=r\}$. For convenience in what follows, we state these conditions of this section below.

(C1) There is a $p>0$ such that $f(t, y)<\eta p$ for $0 \leqslant y \leqslant p$ and $v-2 \leqslant t \leqslant v+b$.

(C2) There is a $p>0$ such that $f(t, y)>\lambda p$ for $\gamma p \leqslant y \leqslant p$ and $(\nu+b) / 4 \leqslant t \leqslant 3(\nu+b) / 4$.

(C3) $f_{0}>\lambda, f_{\infty}>\lambda$.

(C4) $f^{0}<\eta, f^{\infty}<\eta$.

Lemma 6 (see [8]). Suppose that there exist two different positive numbers $r$ and $R$ such that $f$ satisfies condition $(C 1)$ at $r$ and condition (C2) at R. Then FBVP (1) has at least one positive solution $y_{0} \in \mathscr{K}$ satisfying $\min \{r, R\} \leqslant\left\|y_{0}\right\| \leqslant$ $\max \{r, R\}$.

Theorem 7. Assume that $f$ satisfies conditions (C1) and (C3). Then FBVP (1) has at least two positive solutions $y_{1}$ and $y_{2}$ with $0<\left\|y_{1}\right\|<p \leqslant\left\|y_{2}\right\|$.

Proof. Suppose that (C3) holds. Since $f_{0}>\lambda$, there exist $\varepsilon>0$ and $0<r_{0}<p$ such that $f(t, y) \geqslant(\lambda+\varepsilon) y, 0 \leqslant y \leqslant r_{0}$, $t \in[\nu-2, \nu+b]_{\mathbb{N}-2}$. Let $r_{1} \in\left(0, r_{0}\right)$ and note that $[(b-v) / 2]+v \in$ $[(b+v) / 4,3(b+v) / 4]$. Thus for $y \in \partial \Omega_{r_{1}}$, we get

$$
\begin{aligned}
& (T y)\left(\left[\frac{b-v}{2}\right]+v\right) \\
& =\sum_{s=0}^{b+1} G\left(\left[\frac{b-v}{2}\right]+v, s\right) f(s+v-1, y(s+v-1)) \\
& \geqslant \sum_{s=0}^{b+1} G\left(\left[\frac{b-v}{2}\right]+v, s\right)(\lambda+\varepsilon) y
\end{aligned}
$$

$$
\begin{aligned}
& \geqslant(\lambda+\varepsilon) \cdot \gamma\|y\| \sum_{s=[((\nu+b) / 4)-v+1]}^{[(3(\nu+b) / 4)-v+1]} G\left(\left[\frac{b-v}{2}\right]+\nu, s\right) \\
& >\lambda \cdot \gamma\|y\| \sum_{s=[((\nu+b) / 4)-v+1]}^{[(3(\nu+b) / 4)-v+1]} G\left(\left[\frac{b-v}{2}\right]+v, s\right) \\
& =r_{1}
\end{aligned}
$$

that is, there is $\|T y\|>\|y\|$ for $y \in \mathscr{K} \cap \partial \Omega_{r_{1}}$.

On the other hand, since $f_{\infty}>\lambda$, there exist $\sigma>0$ and $R_{0}>0$ such that $f(t, y) \geqslant(\lambda+\sigma) y, y \geqslant R_{0}, t \in[\nu-2, \nu+$ $b]_{\mathbb{N}_{\nu^{2}}}$. Choose $R_{1}>\max \left\{(1 / \gamma) R_{0}\right.$, p . If $y \in \partial \Omega_{R_{1}}$, then $y(t) \geqslant$ $\gamma\|y\|^{2}>R_{0}$ for $(\nu+b) / 4 \leqslant t \leqslant 3(\nu+b) / 4$. So it follows that

$$
\begin{aligned}
& (T y)\left(\left[\frac{b-v}{2}\right]+v\right) \\
& =\sum_{s=0}^{b+1} G\left(\left[\frac{b-v}{2}\right]+v, s\right) f(s+v-1, y(s+v-1)) \\
& \geqslant \sum_{s=0}^{b+1} G\left(\left[\frac{b-v}{2}\right]+\nu, s\right)(\lambda+\sigma) y \\
& \geqslant(\lambda+\sigma) \cdot \gamma\|y\| \sum_{s=[((\nu+b) / 4)-v+1]}^{[(3(\nu+b) / 4)-v+1]} G\left(\left[\frac{b-v}{2}\right]+\nu, s\right) \\
& >\lambda \cdot \gamma\|y\| \sum_{s=[((\nu+b) / 4)-v+1]}^{[(3(\nu+b) / 4)-v+1]} G\left(\left[\frac{b-v}{2}\right]+v, s\right) \\
& =R_{1} \text {, }
\end{aligned}
$$

from which we see that $\|T y\|>\|y\|$ for $y \in \mathscr{K} \cap \partial \Omega_{R_{1}}$.

For any $y \in \partial \Omega_{p}$, from $(C 1)$, we have $f(t, y) \leqslant \eta p, t \in$ $[v-2, v+b]_{\mathbb{N}_{\gamma-2}}$.

Consider

$$
\begin{aligned}
(T y)(t) & =\sum_{s=0}^{b+1} G(t, s) f(s+v-1, y(s+v-1)) \\
& \leqslant \sum_{s=0}^{b+1} G(s+v-1, s) \eta p \\
& =p=\|y\| ;
\end{aligned}
$$

that is, there is $\|T y\| \leqslant\|y\|$ for $y \in \mathscr{K} \cap \partial \Omega_{p}$.

Consequently, Lemma 5 implies that there are two fixed points $y_{1}$ and $y_{2}$ of operator $T$ such that $0<\left\|y_{1}\right\|<p<\left\|y_{2}\right\|$. And this completes the proof.

Remark 8. By the proof of Theorem 7, we know that the conclusion of Theorem 7 is valid if $(C 3)$ is replaced by $f_{0}=$ $+\infty$ and $f_{\infty}=+\infty$. Namely, our result in this paper improve Theorem 3.4 in [8].

Theorem 9. Suppose that conditions (C2) and (C4) hold, $f>$ 0 for $t \in[v-2, v+b]_{\mathbb{N}_{v-2}}$. Then FBVP (1) has at least two positive solutions $y_{1}$ and $y_{2}$ with $0<\left\|y_{1}\right\|<p<\left\|y_{2}\right\|$. 
Proof. From the assumption $f^{0}<\eta$, one can find $\varepsilon>0(\varepsilon<\eta)$ and $0<r_{0}<p$ such that $f(t, y) \leqslant(\eta-\varepsilon) y, 0 \leqslant y \leqslant r_{0}$, $t \in[v-2, v+b]_{\mathbb{N}_{v-2}}$. Let $r_{2} \in\left(0, r_{0}\right)$; then for $y \in \partial \Omega_{r_{2}}$, we have

$$
\begin{aligned}
(T y)(t) & =\sum_{s=0}^{b+1} G(t, s) f(s+v-1, y(s+v-1)) \\
& \leqslant \sum_{s=0}^{b+1} G(s+v-1, s)(\eta-\varepsilon) r_{2} \\
& <\eta r_{2} \sum_{s=0}^{b+1} G(s+v-1, s) \\
& =r_{2}=\|y\|
\end{aligned}
$$

from which we see that $\|T y\|<\|y\|$ for $y \in \partial \Omega_{r_{2}}$.

On the other hand, since $f^{\infty}<\eta$, there exist $0<\sigma<\eta$ and $R_{0}>0$ such that

$$
f(t, y) \leqslant \sigma y, \quad y \geqslant R_{0}, \quad t \in[v-2, v+b]_{\mathbb{N}_{\nu-2}} .
$$

Denote $M=\max _{(t, y) \in[\nu-2, v+b]_{\mathbb{N}_{\nu-2}} \times\left[0, R_{0}\right]} f(t, y)$; then $0 \leqslant$ $f(t, y) \leqslant \sigma y+M, 0 \leqslant y<+\infty$. Let $R_{2}>\max \{2 p, M /(\eta-\sigma)\}$. For $y \in \partial \Omega_{R_{2}}$, we have

$$
\begin{aligned}
\|T y\| & =\max _{t \in[\nu-2, \nu+b]_{N_{\nu-2}}} \sum_{s=0}^{b+1} G(t, s) f(s+v-1, y(s+v-1)) \\
& \leqslant \sum_{s=0}^{b+1} G(s+v-1, s) f(s+v-1, y(s+v-1)) \\
& \leqslant(\sigma\|y\|+M) \sum_{s=0}^{b+1} G(s+v-1, s) \\
& =\left(\sigma R_{2}+M\right) \cdot \frac{1}{\eta}<R_{2} .
\end{aligned}
$$

Therefore, we have $\|T y\|<\|y\|$ for $y \in \partial \Omega_{R_{2}}$.

Finally, for any $y \in \partial \Omega_{p}$, since $\gamma p \leqslant y(t) \leqslant p$ for $t \in$ $[(\nu+b) / 4,3(\nu+b) / 4]$, we estimate

$$
\begin{aligned}
& (T y)\left(\left[\frac{b-v}{2}\right]+v\right) \\
& =\sum_{s=0}^{b+1} G\left(\left[\frac{b-v}{2}\right]+v, s\right) f(s+v-1, y(s+v-1)) \\
& >\lambda \gamma p \sum_{s=[((\nu+b) / 4)-v+1]}^{[(3(\nu+b) / 4)-v+1]} G\left(\left[\frac{b-v}{2}\right]+v, s\right) \\
& =p=\|y\| .
\end{aligned}
$$

Hence $\|T y\|>\|y\|$ for $y \in \mathscr{K} \cap \partial \Omega_{p}$.

By Lemma 5, the proof is complete.
Remark 10. From the proof of Theorem 9, we know that the conclusion of Theorem 9 is valid if the condition (C4) is replaced by $f^{0}=0$ and $f^{\infty}=0$.

Remark 11. Theorem 9 is not included in $[6,8]$.

From the proof of Theorems 7 and 9, we have the following.

Theorem 12. Suppose that $f_{0}>\lambda$, $f^{\infty}<\eta$. Then FBVP (1) has at least one positive solution.

Theorem 13. Suppose that $f^{0}<\eta, f_{\infty}>\lambda$. Then FBVP (1) has at least one positive solution.

Remark 14. Theorems 12 and 13 in this paper significantly generalize Theorems 4.1 and 4.2 in [6].

Example 15. Consider the following boundary value problems:

$$
\begin{aligned}
\triangle^{9 / 8} y(t) & =-\frac{1}{100} e^{t-(57 / 8)}\left(y^{1 / 2}\left(t+\frac{1}{8}\right)+\frac{1}{4} y^{2}\left(t+\frac{1}{8}\right)\right), \\
y\left(-\frac{7}{8}\right) & =y\left(\frac{65}{8}\right)=0,
\end{aligned}
$$

where $v=9 / 8$ and $b=6$, and $f(t, y)=$ $(1 / 100) e^{t-(29 / 4)}\left(y^{1 / 2}+(1 / 4) y^{2}\right)$. A simple computation shows that $\eta>0.0126, \lambda=5 / 18 \gamma(\gamma$ is the constant in Lemma 3(iii)), and $f_{0}=f_{\infty}=+\infty$. Taking $p=1$, we get

$$
\begin{aligned}
f(t, y) & =\frac{1}{100} e^{t-(29 / 4)}\left(y^{1 / 2}+\frac{1}{4} y^{2}\right) \\
& \leqslant \frac{1}{100}\left(p^{1 / 2}+\frac{1}{4} p^{2}\right) \\
& =0.0125<\eta p,
\end{aligned}
$$

$0 \leqslant y \leqslant p, v-2 \leqslant t \leqslant b+v$. All conditions in Theorem 7 are satisfied. Therefore FBVP (20) has at least two positive solutions $y_{1}$ and $y_{2}$ such that $0<\left\|y_{1}\right\|<1<\left\|y_{2}\right\|$.

Example 16. Consider the following boundary value problems:

$$
\begin{aligned}
\Delta^{9 / 8} y(t) & =-\frac{1}{100} y\left(t+\frac{1}{8}\right)\left[1+\frac{28-\gamma}{\gamma\left(1+y^{2}(t+(1 / 8))\right)}\right], \\
y\left(-\frac{7}{8}\right) & =y\left(\frac{65}{8}\right)=0,
\end{aligned}
$$


where $\nu=9 / 8$ and $b=6$, and $f(t, y)=(1 / 100) y[1+((28-$ $\left.\left.\gamma) / \gamma\left(1+y^{2}\right)\right)\right](\gamma$ is the constant in Lemma 3(iii)); it is easy to compute that

$$
\begin{aligned}
f^{\infty} & =\limsup _{y \rightarrow+\infty} \max _{t \in[\nu-2, \nu+b]_{N_{\nu-2}}} \frac{1}{100}\left(1+\frac{28-\gamma}{\gamma\left(1+y^{2}\right)}\right) \\
& =\frac{1}{100}<0.0126<\eta, \\
f_{0} & =\liminf _{y \rightarrow 0^{+}} \min _{t \in[\nu-2, \nu+b]_{N_{\nu-2}}} \frac{1}{100}\left(1+\frac{28-\gamma}{\gamma\left(1+y^{2}\right)}\right) \\
& =\frac{7}{25 \gamma}>\frac{5}{18 \gamma}=\lambda,
\end{aligned}
$$

which yields the condition of Theorem 12. By Theorem 12, FBVP (22) has at least one positive solution.

\section{Existence of Solutions}

In this section, we give the existence of solutions for problem (1). We will prove this result by using Schauder's fixed point theorem and provide an example to illustrate our results.

Theorem 17. Let $f:[v-1, v+b]_{\mathbb{N}_{v-1}} \times \mathbb{R} \rightarrow \mathbb{R}$ be a continuous function. Suppose that one of the following conditions is satisfied.

$\left(H_{1}\right)$ There exist a nonnegative function $a(t) \in \mathscr{C}[v-1, v+b]$ and a constant $c$ such that $|f(t, y)| \leqslant a(t)+c|y|^{\rho}$, where $c \geqslant 0,0<\rho<1$.

$\left(H_{2}\right)|f(t, y)| \leqslant c|y|^{\rho}$, where $c>0, \rho>1$.

Then problem (1) has at least one solution.

Proof. First, suppose the condition $\left(H_{1}\right)$ can be satisfied. Let

$$
E=\left\{y(t) \in \mathscr{B}:\|y\| \leqslant R, t \in[\nu-2, v+b]_{\mathbb{N}_{\nu-2}}\right\},
$$

where

$$
\begin{aligned}
& R \geqslant \max \left\{2 \sum_{s=0}^{b+1} G(s+\nu-1, s)|a(s+\nu-1)|,\right. \\
& {\left.\left[4 c \frac{\Gamma(\nu+b+2)}{\Gamma(b+2) \Gamma(\nu+1)}\right]^{1 /(1-\rho)}\right\} . }
\end{aligned}
$$

Obviously, $E$ is a closed ball in the Banach space $\mathscr{B}$.

Now we prove that $T: E \rightarrow E$. For any $y \in E$, then

$$
\begin{aligned}
|(T y)(t)| \leqslant \sum_{s=0}^{b+1} \mid G(t, s)[ & a(s+v-1) \\
& \left.+c|y(s+v-1)|^{\rho}\right] \mid
\end{aligned}
$$

$$
\begin{aligned}
& \leqslant \sum_{s=0}^{b+1}|G(t, s) a(s+v-1)| \\
& +\sum_{s=0}^{b+1} c\|y(s+v-1)\|^{\rho} \cdot|G(t, s)| \\
& \leqslant \sum_{s=0}^{b+1} G(s+v-1, s)|a(s+v-1)| \\
& +c R^{\rho}\left(\sum_{s=0}^{t-v}|G(t, s)|+\sum_{s=t-v+1}^{b+1}|G(t, s)|\right) \\
& \leqslant \sum_{s=0}^{b+1} G(s+v-1, s)|a(s+v-1)| \\
& +\frac{c R^{\rho}}{\Gamma(\nu)}\left(\sum_{s=0}^{t-\nu}(t-s-1)^{\frac{\nu-1}{2}}\right. \\
& \left.+\sum_{s=0}^{b+1} \frac{t^{\frac{\nu-1}{}}(\nu+b-s)^{\frac{\nu-1}{}}}{(\nu+b+1)^{\frac{\nu-1}{}}}\right) \\
& \leqslant \sum_{s=0}^{b+1} G(s+v-1, s)|a(s+v-1)| \\
& +c R^{\rho} \max _{t \in[\nu-2, \nu+b]_{N_{\nu-2}}}\left(\frac{1}{\Gamma(\nu)} \sum_{s=0}^{t-v}(t-s-1)^{\frac{\nu-1}{}}\right)
\end{aligned}
$$

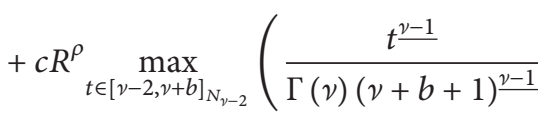

$$
\begin{aligned}
& \left.\times \sum_{s=0}^{b+1}(\nu+b-s)^{\frac{\nu-1}{}}\right) .
\end{aligned}
$$

Notice that

$$
\begin{aligned}
\frac{1}{\Gamma(\nu)} \sum_{s=0}^{t-\nu}(t-s-1)^{\frac{\nu-1}{}} \\
=\frac{1}{\Gamma(\nu)}\left[-\frac{1}{\nu}(t-s)^{\underline{\nu}}\right]_{s=0}^{t-\nu+1} \\
=\frac{1}{\Gamma(\nu+1)} t^{\underline{\nu}} \leq \frac{\Gamma(\nu+b+1)}{\Gamma(b+1) \Gamma(\nu+1)}, \\
\leqslant \frac{1}{\Gamma(\nu)} \sum_{s=0}^{b+1}(\nu+b-s)^{\frac{\nu-1}{}} \\
=\frac{1}{\Gamma(\nu)}\left[-\frac{1}{\nu}(\nu+b-s+1)^{\underline{v}}\right]_{s=0}^{b+2}(\nu+b-s)^{\frac{\nu-1}{}} \\
=\frac{\Gamma(\nu+b+2)}{\Gamma(b+2) \Gamma(\nu+1)} .
\end{aligned}
$$


Thus

$$
\begin{aligned}
& |(T y)(t)| \\
& \leqslant \sum_{s=0}^{b+1} G(s+v-1, s)|a(s+v-1)| \\
& \quad+2 c R^{\rho} \frac{\Gamma(\nu+b+2)}{\Gamma(b+2) \Gamma(v+1)} \\
& \quad \leqslant \frac{R}{2}+\frac{R}{2}=R
\end{aligned}
$$

we get $\|T y\| \leqslant R$. choose

Second, suppose the condition $\left(H_{2}\right)$ can be satisfied; we

$$
0<R \leqslant\left(\frac{\Gamma(b+2) \Gamma(\nu+1)}{2 c \Gamma(\nu+b+2)}\right)^{1 /(\rho-1)} .
$$

With a similar argument as the above, we obtain $\|T y\| \leqslant R$. Consequently, we get $T: E \rightarrow E$.

Note that $T$ is a summation operator on a discrete finite set. Hence, $T$ is trivially completely continuous. Therefore, according to the Schauder fixed point theorem, $T$ has a fixed point $y$. Namely, $y$ is a solution of problem (1). The theorem is proved.

Remark 18. In this section, $f$ is only a continuous function, without nonnegative assumptions on function $f$.

Remark 19. If $\rho=1$ in $\left(H_{1}\right)$, we need the condition $c(\Gamma(\nu+$ $b+2) / \Gamma(b+2) \Gamma(\nu+1)) \leqslant 1 / 4$. Then, choose

$$
R \geqslant 2 \sum_{s=0}^{b} G(s+v-1, s)|a(s+v-1)| .
$$

If $\rho=1$ in $\left(H_{2}\right)$, we only need the condition $c(\Gamma(\nu+b+2) / \Gamma(b+$ 2) $\Gamma(\nu+1)) \leqslant 1 / 2$. Then the conclusion of Theorem 17 remains true.

Example 20. Consider the fractional difference equation

$$
\begin{aligned}
\triangle^{3 / 2} y(t) & =-\left(t+\frac{1}{2}\right)^{4} y^{\rho}\left(t+\frac{1}{2}\right), \\
y\left(-\frac{1}{2}\right) & =y\left(\frac{5}{2}+b\right)=0,
\end{aligned}
$$

where $t \in[v-2, v+b]_{\mathbb{N}_{v-2}}, 0<\rho<1$ or $\rho>1, b \in \mathbb{N}_{+}$, and $a(t)=0, f(t, y)=t^{4} y^{\rho}$. By using Theorem 17, the existence of solutions is obvious for $0<\rho<1$ or $\rho>1$.

\section{Conflict of Interests}

The authors declare that there is no conflict of interests regarding the publication of this paper.

\section{Acknowledgments}

The authors are very grateful to the reviewers for their valuable suggestions and useful comments, which led to the improvement of this paper. Project supported by the National Natural Science Foundation of China (Grant no. 11271235) and Shanxi Datong University Institute (2009-Y-15, 2010B-01, 2013K5) and the Development Foundation of Higher Education Department of Shanxi Province (20111020).

\section{References}

[1] J. B. Díaz and T. J. Osler, "Differences of fractional order," Mathematics of Computation, vol. 28, pp. 185-202, 1974.

[2] H. L. Gray and N. F. Zhang, "On a new definition of the fractional difference," Mathematics of Computation, vol. 50, no. 182, pp. 513-529, 1988.

[3] F. M. Atici and P. W. Eloe, "A transform method in discrete fractional calculus," International Journal of Difference Equations, vol. 2, no. 2, pp. 165-176, 2007.

[4] F. M. Atici and P. W. Eloe, "Initial value problems in discrete fractional calculus," Proceedings of the American Mathematical Society, vol. 137, no. 3, pp. 981-989, 2009.

[5] F. M. Atıcı and S. Şengül, "Modeling with fractional difference equations," Journal of Mathematical Analysis and Applications, vol. 369, no. 1, pp. 1-9, 2010.

[6] F. M. Atıc1 and P. W. Eloe, "Two-point boundary value problems for finite fractional difference equations," Journal of Difference Equations and Applications, vol. 17, no. 4, pp. 445-456, 2011.

[7] C. S. Goodrich, "On nonlinear boundary conditions satisfying certain asymptotic behavior," Nonlinear Analysis: Theory, Methods \& Applications, vol. 76, pp. 58-67, 2013.

[8] C. S. Goodrich, "Some new existence results for fractional difference equations," International Journal of Dynamical Systems and Differential Equations, vol. 3, no. 1-2, pp. 145-162, 2011.

[9] C. S. Goodrich, "On a fractional boundary value problem with fractional boundary conditions," Applied Mathematics Letters, vol. 25, no. 8, pp. 1101-1105, 2012.

[10] C. S. Goodrich, "On a discrete fractional three-point boundary value problem," Journal of Difference Equations and Applications, vol. 18, no. 3, pp. 397-415, 2012.

[11] C. S. Goodrich, "Continuity of solutions to discrete fractional initial value problems," Computers \& Mathematics with Applications, vol. 59, no. 11, pp. 3489-3499, 2010.

[12] C. S. Goodrich, "Nonlocal systems of BVPs with asymptotically sublinear boundary conditions," Applicable Analysis and Discrete Mathematics, vol. 6, no. 2, pp. 174-193, 2012.

[13] M. Holm, "Sum and difference compositions in discrete fractional calculus," Cubo, vol. 13, no. 3, pp. 153-184, 2011.

[14] G.-C. Wu and D. Baleanu, "New applications of the variational iteration method-from differential equations to $q$-fractional difference equations," Advances in Difference Equations, vol. 2013, article 21, 2013.

[15] S. G. Kang, X. H. Zhao, and H. Q. Chen, "Positive solutions for boundary value problems of fractional difference equations depending on parameters," Advances in Difference Equations, vol. 2013, article 376, 2013.

[16] S. G. Kang, Y. Li, and H. Q. Chen, "Positive solutions to boundary value problem of fractional difference equation with nonlocal conditions," Advances in Difference Equations, vol. 2014, article 7, 2014.

[17] R. P. Agarwal, M. Meehan, and D. O'Regan, Fixed Point Theory and Applications, Cambridge University Press, Cambridge, UK, 2001. 


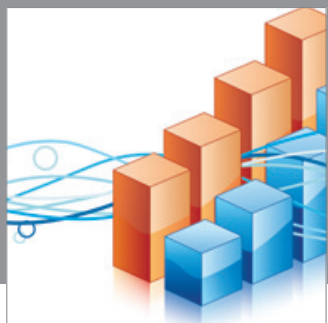

Advances in

Operations Research

mansans

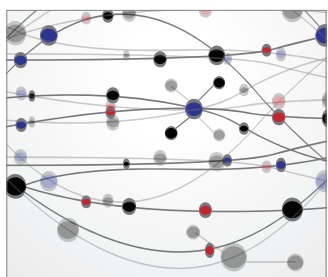

The Scientific World Journal
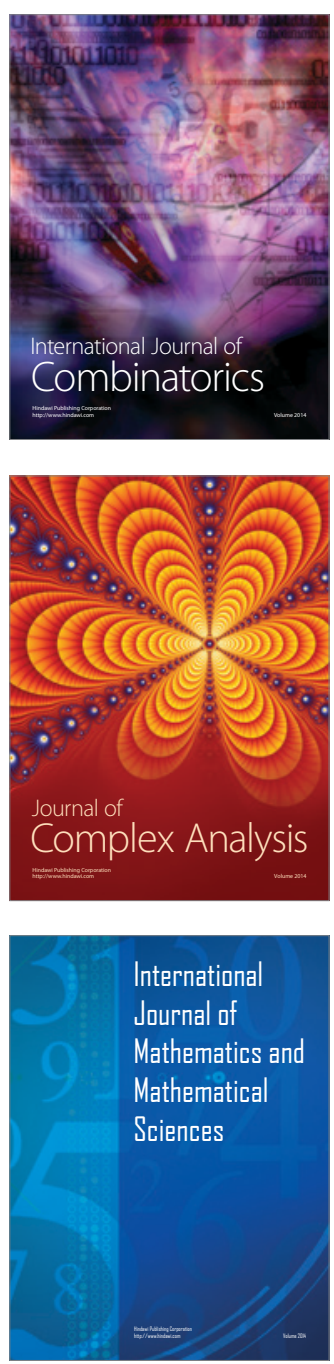
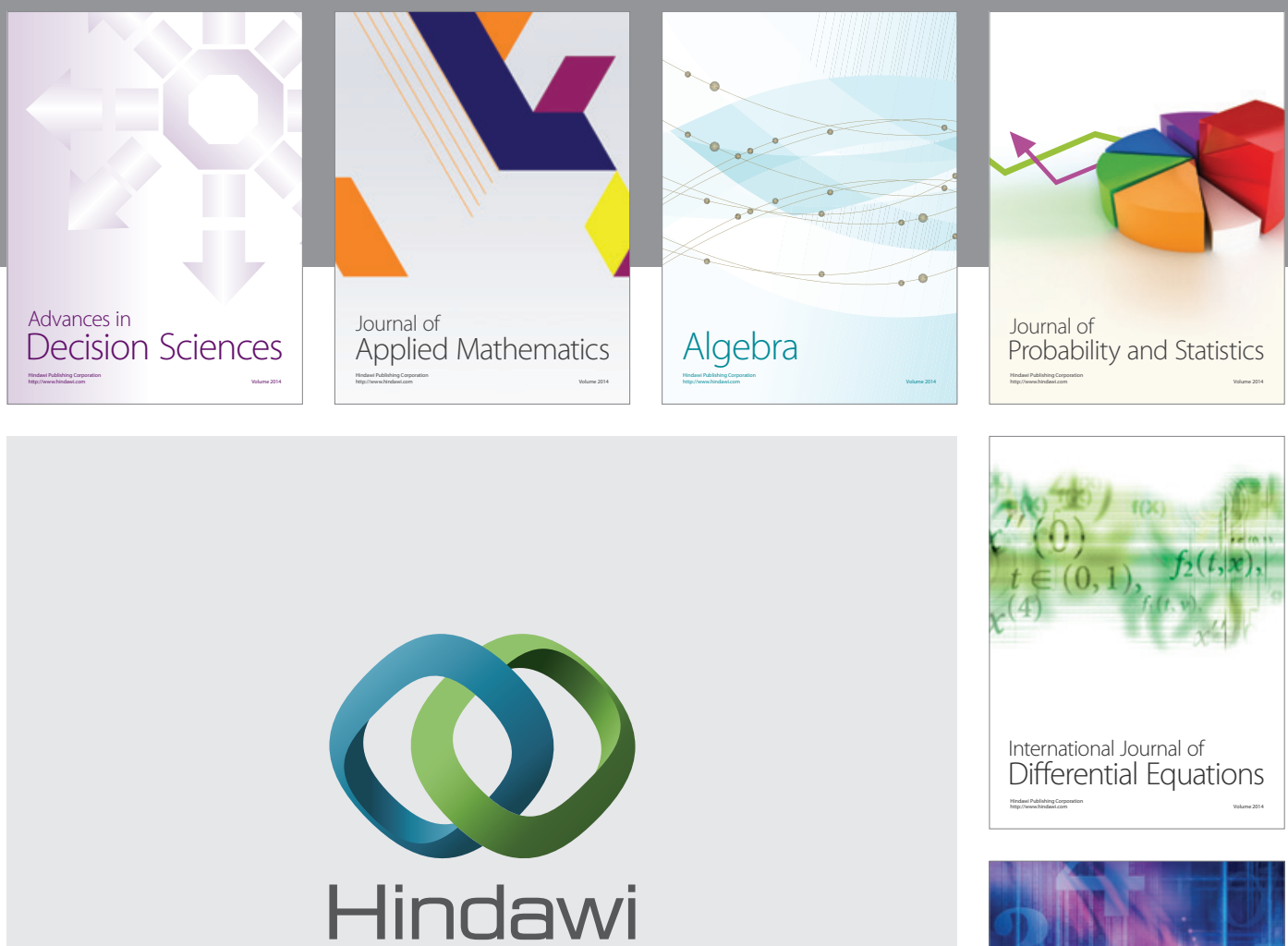

Submit your manuscripts at http://www.hindawi.com
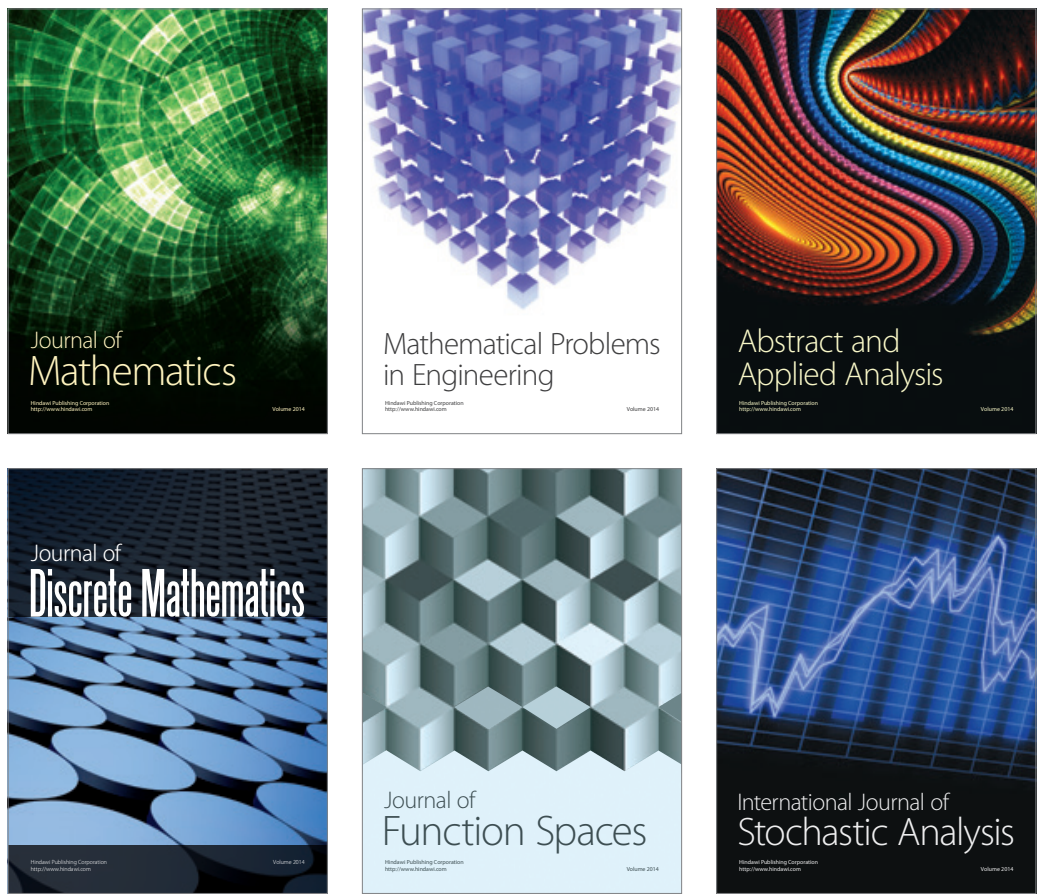

Journal of

Function Spaces

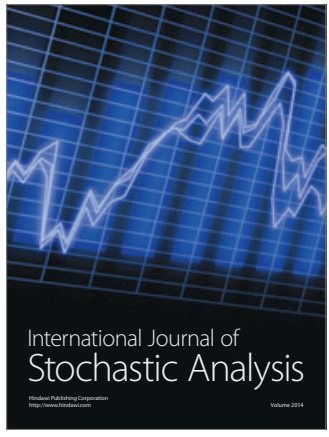

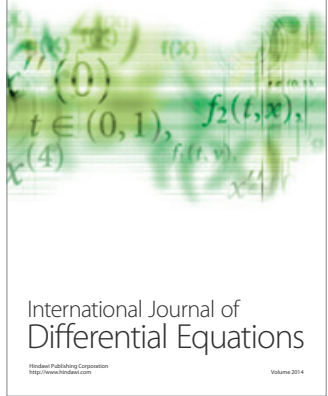
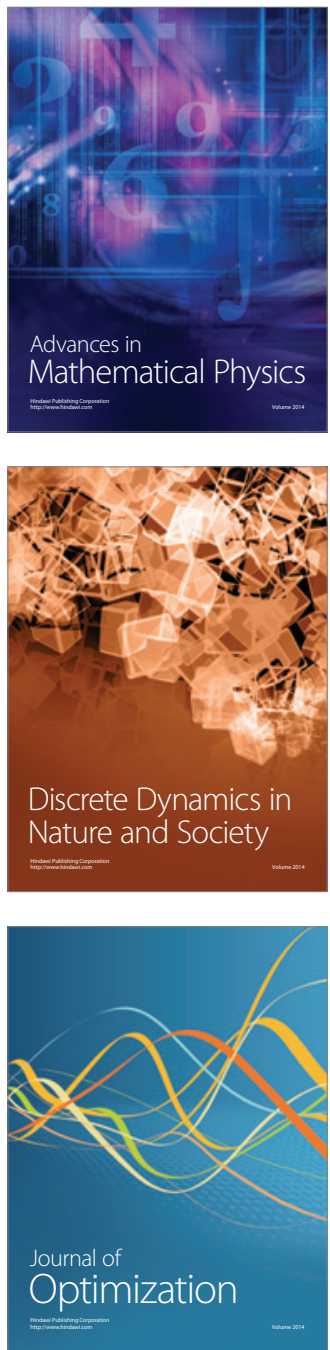